\title{
Integrin- $\alpha 7$ expression positively correlates with CD44 and CD133 expressions, and its high expression associates with advanced tumor features as well as poor survivals in non-small cell lung cancer patients
}

\author{
Xiaowu Shi, Fangjing Wen, Guoxing Xu, Yi Hu \\ Department of Respiratory Medicine, The Central Hospital of Wuhan, Tongji Medical College, Huazhong University of Science and Technology, \\ Wuhan 430014, China \\ Contributions: (I) Conception and design: X Shi; (II) Administrative support: X Shi; (III) Provision of study materials or patients: All authors; (IV) \\ Collection and assembly of data: All authors; (V) Data analysis and interpretation: F Wen, G Xu; (VI) Manuscript writing: All authors; (VII) Final \\ approval of manuscript: All authors. \\ Correspondence to: Yi Hu. Department of Respiratory Medicine, The Central Hospital of Wuhan, Tongji Medical College, Huazhong University of \\ Science and Technology, 26 Shengli Street, Jiang'an District, Wuhan 430014, China. Email: heqiao424297@163.com.
}

\begin{abstract}
Background: This study aimed to explore the correlation of integrin- $\alpha 7$ (ITGA7) with common cancer stem cell marker (CD44 and CD133) expressions, clinicopathological characteristics and survival profiles in non-small cell lung cancer (NSCLC) patients.

Methods: Two hundred and seventy NSCLC patients who underwent resection were reviewed in this study and the expressions of ITGA7, CD44 and CD133 were detected using immunohistochemistry (IHC) assay in tumor tissue specimens. The clinical data (including age, gender, pathological grade, tumor size, lymph node metastasis (LYN), and TNM stage), survival data [including disease-free survival (DFS) and overall survival (OS)] were collected.

Results: The numbers of NSCLC patients with ITGA7 high expression, CD44 high expression and CD133 high expression were 170 (63.0\%), 241 (89.3\%) and 37 (13.7\%) respectively, and ITGA7 expression was positively associated with CD44 expression and CD133 expression. ITGA7 high expression was associated with higher pathological grade, larger tumor size, LYN and elevated TNM stage. However, there was no correlation of ITGA7 expression with age or gender. As for survival, patients with ITGA7 high expression presented reduced DFS and OS compared with those with ITGA7 low expression. In addition, multivariate Cox's proportional hazards regression model analyses exhibited that ITGA7 high expression, higher pathological grade and LYN were independent risk factors for DFS and OS in NSCLC patients.

Conclusions: ITGA7 expression positively correlates with CD44 and CD133 expressions, and its high expression associates with advanced tumor features as well as poor survivals in NSCLC.
\end{abstract}

Keywords: Integrin- $\alpha 7$ (ITGA7); CD44; CD133; tumor features; survivals; non-small cell lung cancer (NSCLC)

Submitted Apr 04, 2019. Accepted for publication Jul 31, 2019.

doi: $10.21037 /$ tcr.2019.08.31

View this article at: http://dx.doi.org/10.21037/tcr.2019.08.31

\section{Introduction}

According to the global cancer statistics, lung cancer is the most commonly diagnosed cancer as well as the leading cause of cancer-related death, which accounts for approximately $11.6 \%$ of all cancer cases and $18.4 \%$ of the total cancer deaths (1). Non-small cell lung cancer (NSCLC), as the major type of lung cancer, contributes to $85 \%$ of the total lung cancer cases and is further classified 
into adenocarcinoma, large cell carcinoma and squamous cell carcinoma subtypes (2). Current common therapies including neoadjuvant chemotherapy, surgical resection, adjuvant chemotherapy etc. are decided based on the tumor stage of NSCLC patients, and targeted therapies using tyrosine kinase inhibitors (TKIs) have become the standard of care and leads to improved clinical outcomes in NSCLC $(3,4)$. However, the 5-year survival rate of all NSCLC patients is still unfavorable mainly due to metastasis, relapse, multi-drug resistance and serious toxicity caused by chemotherapy/radiotherapy $(2,5)$. Therefore, it is essential to discover potential prognostic biomarkers for NSCLC.

Integrins, as transmembrane cell surface receptors involved in cell-matrix adhesion, consist of 18 Alpha $(\alpha)$ as well as 8 Beta $(\beta)$ subunits, and they directly bind to components of the extracellular matrix and trigger a range of cellular responses that are crucial to the initiation, progression as well as metastasis of solid tumors (6). Some integrins regulate the expression of cancer stem markers and associate with advanced pathological grade as well as poor prognosis in several cancers including NSCLC $(5,7,8)$. And integrin- $\alpha 7$ (ITGA7), as one of integrin family members, binds several laminin isoforms, involving in the skeletal muscle integrity (9). Additionally, recent in vitro studies indicate that ITGA7 promotes cell proliferation, migration and invasion but inhibits cell apoptosis by inducing epithelial-mesenchymal transition and activation of FAK/ MAPK/ERK signaling pathway in several tumors $(10,11)$. In clinical studies, ITGA7 is shown to be correlated with advanced tumor features as well as poor survivals in cancers including glioblastoma and esophageal squamous cell carcinoma $(10,11)$. However, no related research exploring the role of ITGA7 in NSCLC development and progression has been reported until now. Thus, we performed this study to investigate the association of ITGA7 expression with common cancer stem cell markers CD44 and CD133 expressions, clinicopathological characteristics as well as survival profiles in NSCLC patients.

\section{Methods}

\section{Patients}

A total of 270 NSCLC patients who underwent resection at our hospital between January 2010 and December 2013 were reviewed in this study. The screening criteria included (I) confirmed diagnosis of primary NSCLC; (II) received resection with well-preserved tumor specimen; (III) clinical data were available and accessible; (IV) follow-up records were complete. Patients were excluded if they underwent neoadjuvant therapy or were complicated with other malignancies. The present study was approved by the Ethics Committee of our hospital with the number "Hospital Ethical Letter (2018) No. 7". Written informed consents or verbal agreements with type recording were obtained from patients or their guardians.

\section{Immunohistochemistry (IHC) assay}

Patients' tumor tissue specimens, which were obtained by surgical resection, fixed with formalin and embedded in paraffin wax, were collected from specimen chamber. The ITGA7, CD44 and CD133 expressions in the tumor tissue were detected by the IHC assay, which was performed briefly as follows: the specimens were cut into $4 \mu \mathrm{m}$ sections, deparaffinized with xylene, rehydrated with ethanol followed by antigen retrieval, and incubated with $\mathrm{H}_{2} \mathrm{O}_{2}$ for blocking endogenous peroxidase, then were blocked using normal goat serum. Subsequently, the tissue sections were incubated at $4{ }^{\circ} \mathrm{C}$ overnight with first antibody. For the ITGA7, Rabbit polyclonal to ITGA7 antibody (1:1,000; Abcam, Cambridge, UK) was used as the first antibody; as for the CD44 and CD133, Rabbit polyclonal to CD44 antibody (1:1,000; Abcam, Cambridge, UK) and Rabbit polyclonal to CD133 antibody (1:200; Abcam) were used as first antibody, respectively. Next day, the tissue sections were incubated with horseradish peroxidaseconjugated goat-anti-rabbit Immunoglobulin G antibody (1:1,000; Abcam). Finally, the sections were stained with diaminobenzidine (DAB) and hematoxylin, then were sealed and observed under a light microscope.

\section{Assessment of ITGA7, CD44 and CD133 expressions}

According to previous studies (12), a semi-quantitative scoring method based on the average intensity and percentage of positively stained tumor cells by IHC were applied to evaluate the expression of ITGA7, CD44 and CD133 in tumor tissue. The intensity of staining was scored as follows: 0 , no staining; 1 , weak staining; 2 , moderate staining; 3 , strong staining (dark brown). The proportion of positively stained tumor cells was scored as follows: $0,0 \%$; $1, \leq 25 \%$; 2, 26-50\%; 3, 51-75\%; 4, 75-100\%. Total score was calculated by multiplying staining intensity score and the proportion score of positively stained tumor cells, and 


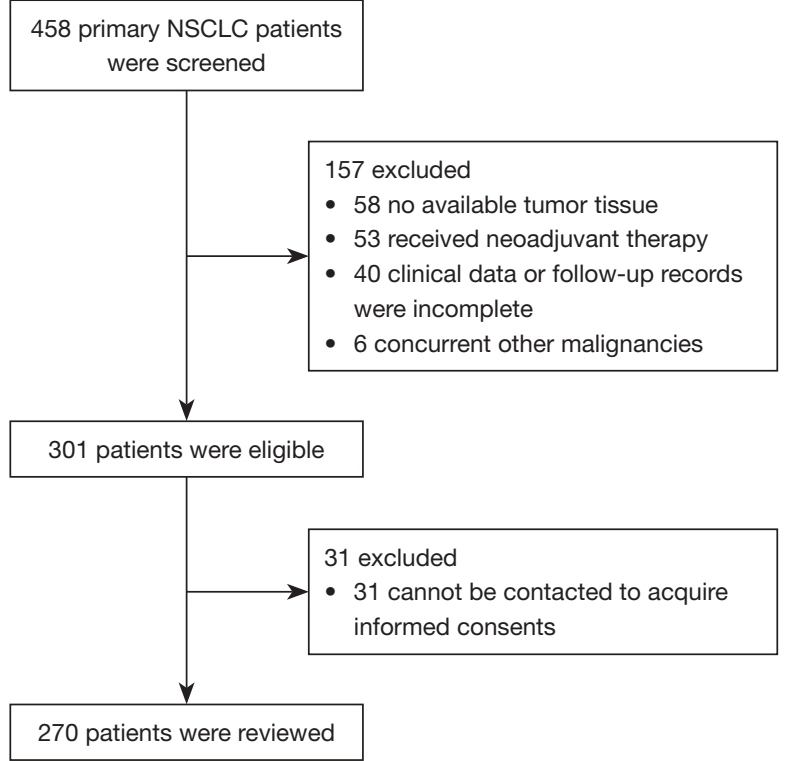

Figure 1 Study flow. NSCLC, non-small cell lung cancer.

the threshold value of 3 points was used to define the high expression and low expression: high expression, the total score $>3$; low expression, the total score $\leq 3$.

\section{Clinical data collection and survival assessment}

Clinical data were collected from electronic medical records, which included age, gender, pathological differentiation, tumor size, lymph node metastasis (LYN), and TNM stage. Moreover, patients' follow-up records were reviewed (last follow-up date was December $31^{\text {st }}, 2018$ ), and the survival data were collected, which were used to calculate the disease-free survival (DFS) and overall survival (OS). The DFS was defined as the duration from resection to disease recurrence or progression or death; the OS was defined as the time interval from resection to death.

\section{Statistical analysis}

All statistical analyses were performed using SPSS 24.0 software (SPSS Inc., Chicago, USA), and graphs were plotted with the use of GraphPad Prism 7.02 (GraphPad Software, La Jolla, USA). Data were displayed as mean value \pm standard deviation and count (percentage). Correlation of ITGA7 with CD44 and CD133 were determined by McNemar's test, and difference of ITGA7 expression among patients with different clinical characteristics was determined by Chi-square test or Wilcoxon rank sum test. The DFS and OS were illustrated by Kaplan-Meier curves, and the differences of DFS and OS between two groups were determined by log-rank test. The factors affecting DFS and OS were examined by univariate and multivariate Cox's proportional hazards regression model analyses. $\mathrm{P}$ value $<0.05$ was considered as significant.

\section{Results}

\section{Study flow}

In the current study, 458 primary NSCLC patients were initially screened, and 157 of them were excluded (including 58 patients without available tumor tissue, 53 patients receiving neoadjuvant therapy, 40 patients without complete clinical data or follow-up records and 6 patients concurrent with other malignancies) (Figure 1). Then 301 patients were further reviewed for eligibility, while 31 of them were excluded because they did not provide informed consents. Finally, the remaining 270 patients were reviewed and included for analysis in this study.

\section{Baseline characteristics}

A total of 270 NSCLC patients with the mean age of $61.9 \pm 10.3$ years were enrolled in this study (Table 1). The number of patients with pathological grade G1, G2 and G3 were $42(15.6 \%), 168(62.2 \%)$ and $60(22.2 \%)$ respectively. The mean tumor size of all patients was $5.5 \pm 2.0 \mathrm{~cm}$, and the number of patients with LYN was $104(38.5 \%)$. As for TNM stage, there were 86 (31.9\%), $86(31.9 \%)$ and 98 (36.3\%) patients with TNM I, II, III stage respectively. The detailed baseline information was shown in Table 1.

\section{Correlation of ITGA7 with CD44 and CD133 in NSCLC patients}

Expressions of ITGA7, CD44 and CD133 in tumor tissue were evaluated by IHC (Figure $2 A$ ). The number of NSCLC patients with ITGA7 high expression, CD44 high expression and CD 133 high expression were 170 (63.0\%), $241(89.3 \%)$ and $37(13.7 \%)$ respectively (Figure $2 B)$. And we further observed that ITGA7 expression was positively associated with CD44 expression $(\mathrm{P}<0.001)$ and $\mathrm{CD} 133$ expression $(\mathrm{P}<0.001)$ (Table 2). 


\section{Correlation of ITGA7 with clinicopathological characteristics in NSCLC patients}

All NSCLC patients were divided into ITGA7 high expression group and ITGA7 low expression group according to the score assessed by a semi-quantitative

Table 1 Characteristics of NSCLC patients

\begin{tabular}{lc}
\hline Characteristics & NSCLC patients (N=270) \\
\hline Age (years), mean \pm SD & $61.9 \pm 10.3$ \\
Gender, No. (\%) & $216(80.0)$ \\
Male & $54(20.0)$ \\
Female & \\
Pathological grade, No. (\%) & $42(15.6)$ \\
G1 & $168(62.2)$ \\
G2 & $60(22.2)$ \\
G3 & $5.5 \pm 2.0$ \\
Tumor size (cm), mean \pm SD & $104(38.5)$ \\
LYN, No. (\%) & \\
TNM stage, No. (\%) & $86(31.9)$ \\
I & $86(31.9)$ \\
II & $98(36.3)$ \\
III
\end{tabular}

NSCLC, non-small cell lung cancer; SD, standard deviation; LYN, lymph node metastasis. scoring method (Table 3). ITGA7 high expression was associated with higher pathological grade $(\mathrm{P}=0.001)$, larger tumor size $(\mathrm{P}=0.029), \mathrm{LYN}(\mathrm{P}=0.014)$ and elevated TNM stage $(\mathrm{P}<0.001)$ in NSCLC patients. However, there was no correlation of ITGA7 expression with age $(\mathrm{P}=0.607)$ or gender $(\mathrm{P}=0.753)$ in NSCLC patients.

\section{Correlation of ITGA7 with survival in NSCLC patients}

DFS in ITGA7 high expression group was shorter compared with ITGA7 low expression group $(\mathrm{P}<0.001)$ (Figure $3 A$ ). And OS in ITGA7 high expression group was also decreased compared with ITGA7 low expression group $(\mathrm{P}<0.001)$ (Figure 3B). These data suggested that ITGA7 expression was negatively correlated with survival in NSCLC patients.

\section{Correlation of ITGA7 and CD44 with survivals in NSCLC patients}

Firstly, we divided all patients into four groups (including: ITGA7 high and CD44 high expression group, ITGA7 high and CD44 low expression group, ITGA7 low and CD44 high expression group, ITGA7 low and CD44 low expression group) and compared the DFS and OS among these four groups. And we found that the DFS $(\mathrm{P}<0.001)$ (Figure $4 A)$ and $\mathrm{OS}(\mathrm{P}<0.001)$ (Figure $4 B$ ) were different among four groups; DFS and OS were both longest in ITGA7 low and CD44 low expression group but the
A

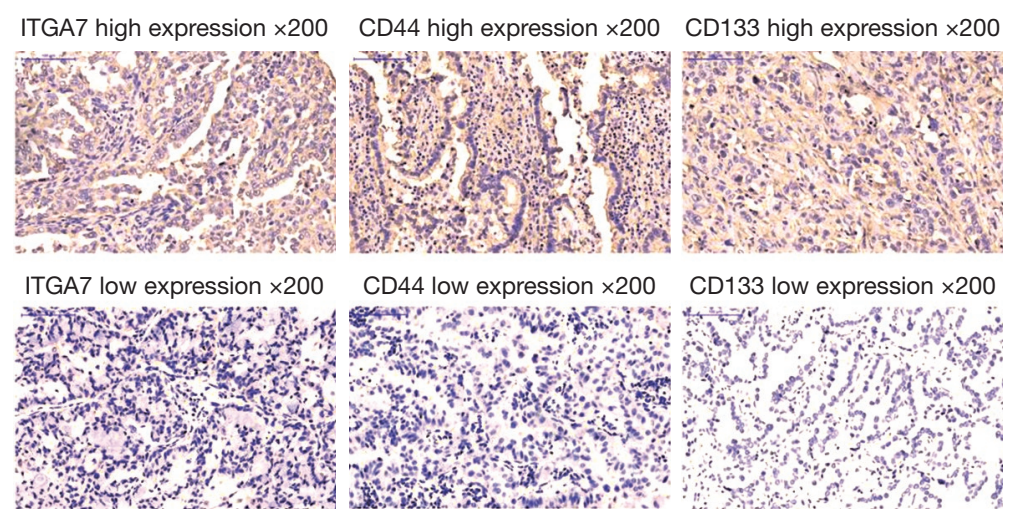

B

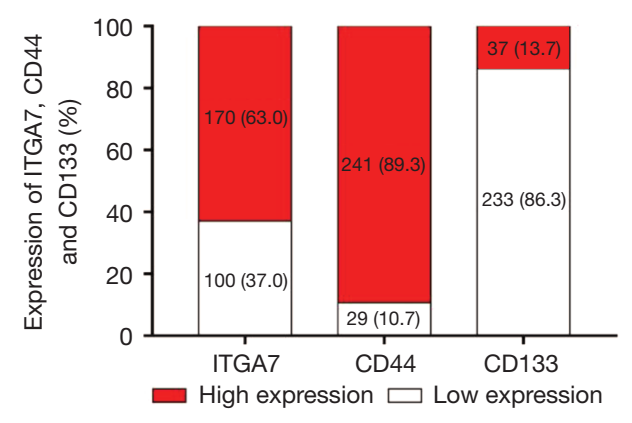

Figure 2 Expression of ITGA7, CD44 and CD133 in NSCLC tumor tissue. Representative IHC images exhibited high and low expression of ITGA7, CD44 as well as CD133. (A) The numbers (percentage) of patients with high and low expression of ITGA7, CD44 and CD133 were presented; (B) data were displayed as count (percentage). ITGA7, integrin- $\alpha$; NSCLC, non-small cell lung cancer; IHC, immunohistochemistry. 
Table 2 Correlations of ITGA7 with CD44 and CD133 expressions

\begin{tabular}{cccccc}
\hline \multirow{2}{*}{ Items } & \multicolumn{2}{c}{ CD44 } & & \multicolumn{2}{c}{ CD133 } \\
\cline { 2 - 3 } \cline { 5 - 5 } & High & Low & & High & Low \\
\hline ITGA7 & & & & \\
High & $159(93.5)$ & $11(6.5)$ & & $33(19.4)$ & $137(80.6)$ \\
Low & $82(82.0)$ & $18(18.0)$ & & $4(4.0)$ & $96(96.0)$ \\
P value & \multicolumn{2}{c}{$<0.001$} & & \multicolumn{2}{c}{$<0.001$} \\
\hline
\end{tabular}

Correlations were determined by McNemar's test, $\mathrm{P}$ value $<0.05$ was considered significant. ITGA7, integrin- $\alpha 7$.

Table 3 Correlations of ITGA7 expression with clinical characteristics

\begin{tabular}{|c|c|c|c|}
\hline \multirow{2}{*}{ Items } & \multicolumn{2}{|c|}{ ITGA7 expression } & \multirow{2}{*}{$P$ value } \\
\hline & High & Low & \\
\hline \multicolumn{3}{|l|}{ Age, No. (\%) } & 0.607 \\
\hline$\leq 60$ years & $82(64.6)$ & $45(35.4)$ & \\
\hline$>60$ years & $88(61.5)$ & $55(38.5)$ & \\
\hline \multicolumn{3}{|c|}{ Gender, No. (\%) } & 0.753 \\
\hline Male & $135(62.5)$ & $81(37.5)$ & \\
\hline Female & $35(64.8)$ & $19(35.2)$ & \\
\hline \multicolumn{3}{|c|}{ Pathological grade, No. (\%) } & 0.001 \\
\hline G1 & $18(42.9)$ & $24(57.1)$ & \\
\hline G2 & $107(63.7)$ & $61(36.3)$ & \\
\hline G3 & $45(75.0)$ & $15(25.0)$ & \\
\hline \multicolumn{3}{|c|}{ Tumor size, No. (\%) } & 0.029 \\
\hline$\leq 5 \mathrm{~cm}$ & $54(54.5)$ & $45(45.5)$ & \\
\hline$>5 \mathrm{~cm}$ & $116(67.8)$ & $55(32.2)$ & \\
\hline \multicolumn{3}{|l|}{ LYN, No. (\%) } & 0.014 \\
\hline No & $95(57.2)$ & $71(42.8)$ & \\
\hline Yes & $75(72.1)$ & $29(27.9)$ & \\
\hline \multicolumn{3}{|c|}{ TNM stage, No. (\%) } & $<0.001$ \\
\hline I & $42(48.8)$ & $44(51.2)$ & \\
\hline II & $55(64.0)$ & $31(36.0)$ & \\
\hline III & $73(74.5)$ & $25(25.5)$ & \\
\hline
\end{tabular}

Correlations were determined by Chi-square test or Wilcoxon rank sum test, $P$ value $<0.05$ was considered significant. ITGA7, integrin- $\alpha 7$; LYN, lymph node metastasis.

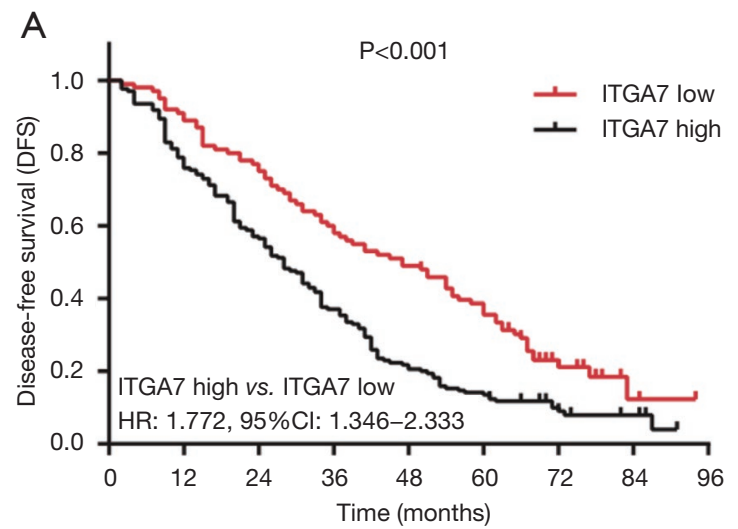

B

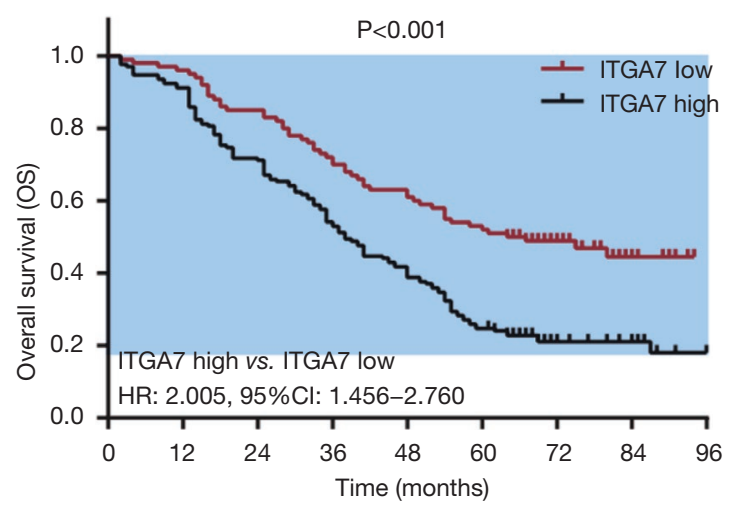

Figure 3 Comparison of survivals between patients with ITGA7 high expression and patients ITGA7 low expression in NSCLC. NSCLC patients with ITGA7 high expression presented shorter DFS compared with those with ITGA7 low expression (A); NSCLC patients with ITGA7 high expression exhibited reduced OS compared with those with ITGA7 low expression (B). The survivals for NSCLC patients were exhibited by Kaplan-Meier curve and the comparison of survival between patients with ITGA7 high expression and patients with ITGA7 low expression was performed by log-rank test. $\mathrm{P}<0.05$ was considered significant. DFS, disease-free survival; OS, overall survival; ITGA7, integrin- $\alpha 7$; HR, hazard ratio; NSCLC, non-small cell lung cancer.

shortest in ITGA7 high and CD44 high expression group. Further univariate Cox's proportional hazards regression model analyses exhibited that ITGA7 high and CD44 high expression group vs. ITGA7 low and CD44 low expression was associated with reduced DFS [HR (hazard ratio): 2.440, 95\% CI: 1.348-4.417] and OS (HR: 2.937, 95\% CI: 

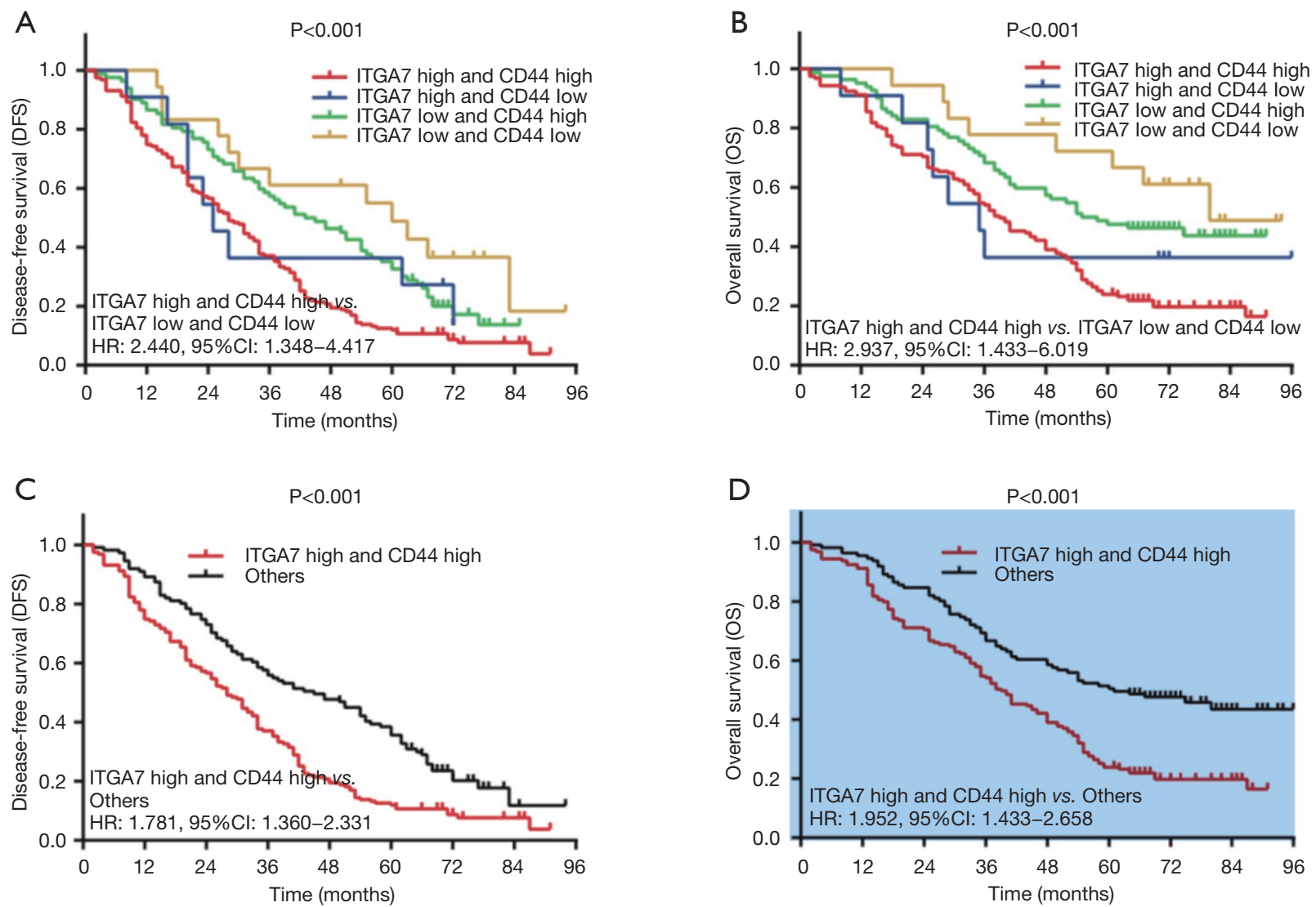

Figure 4 Correlation of ITGA7 and CD44 with DFS and OS in NSCLC patients. Comparison of DFS (A) and OS (B) between ITGA7 high expression and CD44 high expression group, ITGA7 high expression and CD44 low expression group, ITGA7 low expression and CD44 high expression group as well as ITGA7 low expression and CD44 low expression group. Comparison of DFS (C) and OS (D) between ITGA7 high expression and CD44 high expression group with other patients. The survivals for NSCLC patients were exhibited by Kaplan-Meier curve and the comparison of survival between groups were performed by log-rank test. The factors effecting DFS and OS were examined by univariate and multivariate Cox's proportional hazards regression model analyses. $\mathrm{P}<0.05$ was considered significant. DFS, disease-free survival; OS, overall survival; ITGA7, integrin- $\alpha$ 7; HR, hazard ratio; NSCLC, non-small cell lung cancer.

1.433-6.019). Then we divided all patients into two groups (including: ITGA7 high and CD44 high expression group and others) and observed that DFS $(\mathrm{P}<0.001)$ (Figure $4 C)$ as well as $\mathrm{OS}(\mathrm{P}<0.001)$ (Figure $4 D)$ were reduced in ITGA7 high and CD44 high expression group compared with others. Further univariate Cox's proportional hazards regression model analyses showed that ITGA7 high and CD44 high expression group vs. others was associated with reduced DFS (HR:1.781, 95\% CI: 1.360-2.331) and OS (HR: 1.952, 95\% CI: 1.433-2.658). Interestingly, HR values of ITGA7 high and CD44 high expression group vs. ITGA7 low and CD44 low expression group (HR for DFS: 2.440; HR for OS: 2.937) were numerically higher compared with the HR values of ITGA7 high group vs. ITGA7 low expression group (HR for DFS: 1.772; HR for OS: 2.005). These data indicated ITGA7/CD44 combination seemed to present better predicting value for DFS and OS compared to ITGA7 alone.

\section{Correlation of ITGA7 and CD133 with survivals in NSCLC patients}

We divided all patients into four groups (including: ITGA7 high and CD133 high expression group, ITGA7 high and CD133 low expression group, ITGA7 low and CD133 high expression group, ITGA7 low and CD133 low expression group) and compared the DFS and OS among these four groups. And we found that the DFS $(\mathrm{P}<0.001)$ (Figure $5 A$ ) 

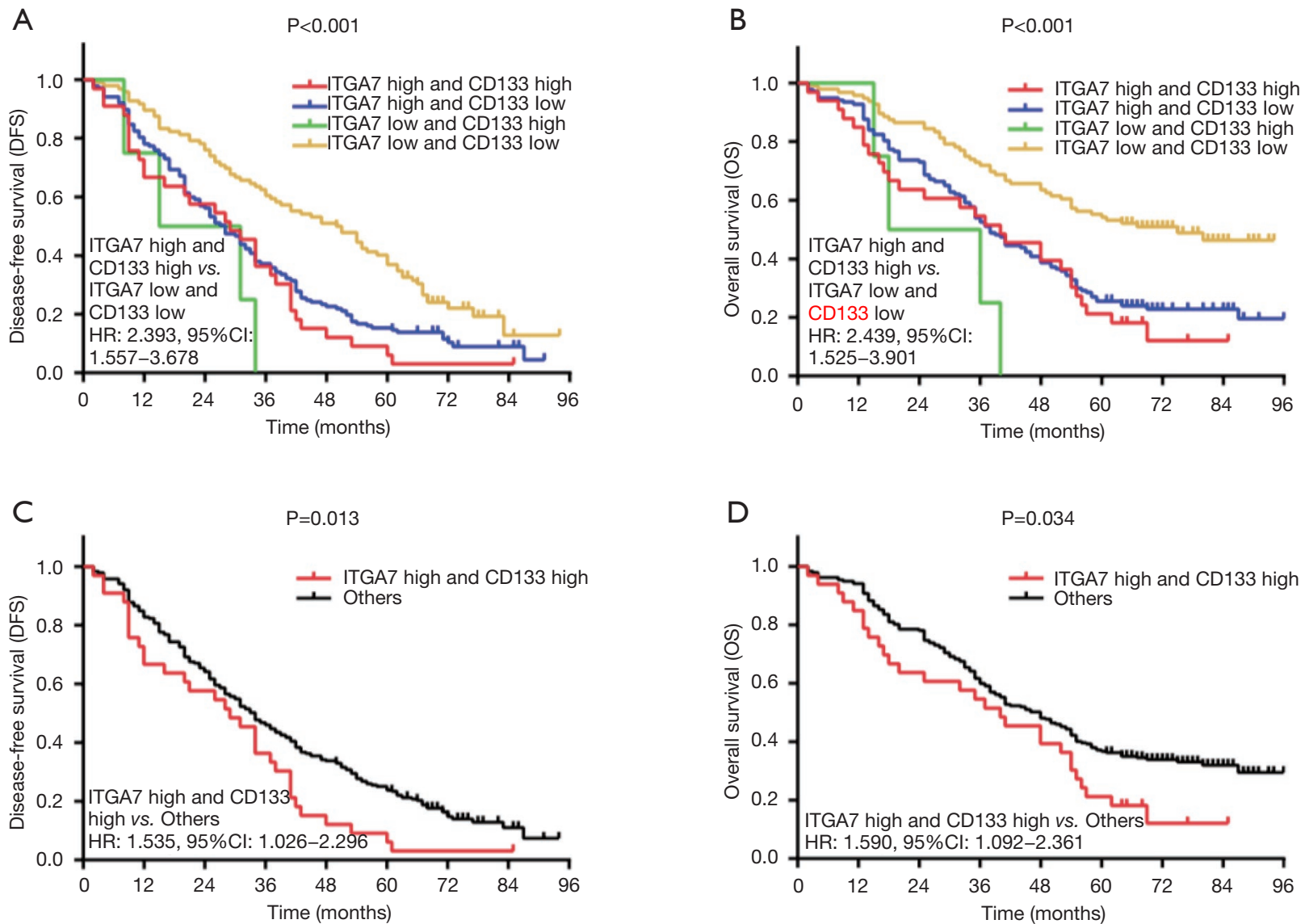

Figure 5 Correlation of ITGA7 and CD133 with DFS and OS in NSCLC patients. Comparison of DFS (A) and OS (B) between ITGA7 high expression and CD133 high expression group, ITGA7 high expression and CD133 low expression group, ITGA7 low expression and CD133 high expression group as well as ITGA7 low expression and CD133 low expression group. Comparison of DFS (C) and OS (D) between ITGA7 high expression and CD133 high expression group with other patients. The survivals for NSCLC patients were exhibited by Kaplan-Meier curve and the comparison of survival between groups were performed by log-rank test. The factors effecting DFS and OS were examined by univariate and multivariate Cox's proportional hazards regression model analyses. $\mathrm{P}<0.05$ was considered significant. DFS, disease-free survival; OS, overall survival; ITGA7, integrin- $\alpha$ 7; HR, hazard ratio; NSCLC, non-small cell lung cancer.

and OS $(\mathrm{P}<0.001)$ (Figure $5 B)$ were different among four groups; DFS and OS were the longest in ITGA7 low and CD133 low expression group but the shortest in ITGA7 high and CD133 high expression group. Further univariate Cox's proportional hazards regression model analyses revealed that ITGA7 high and CD133 high expression group vs. ITGA7 low and CD133 low expression was associated with reduced DFS (HR: 2.393, 95\% CI: $1.557-$ 3.678 ) and OS (HR: 2.439, 95\% CI: $1.525-3.901)$. And then we divided all patients into two groups (including: ITGA7 high and CD133 high expression group and others) and observed that DFS $(\mathrm{P}=0.013)$ (Figure 5C) as well as OS $(\mathrm{P}=0.034)$ (Figure $5 D)$ were reduced in ITGA7 high and CD133 high expression group compared with others. Further univariate Cox's proportional hazards regression model analyses showed that ITGA7 high and CD133 high expression group $v s$. others was associated with decreased DFS (HR: 1.535, 95\% CI: 1.026-2.296) and OS (HR: 1.590, 95\% CI: 1.092-2.361). Importantly, HR values of ITGA7 high and CD133 high expression group vs. ITGA7 low and CD133 low expression group (HR for DFS: 2.393; HR for OS: 2.439) were numerically higher compared with the HR values of ITGA7 high group vs. ITGA7 low expression group (HR for DFS: 1.772; HR for OS: 2.005), while HR values of ITGA7 high and CD133 high expression group vs. others (HR for DFS: 1.535; HR for OS: 1.590) were 
Table 4 Univariate and multivariate Cox's proportional hazard model regression analyses of factors affecting DFS

\begin{tabular}{|c|c|c|c|c|}
\hline Items & \multicolumn{2}{|c|}{ Univariate Cox's regression } & \multicolumn{2}{|c|}{ Multivariate Cox's regression } \\
\hline ITGA7 expression (high vs. low) & $<0.001$ & $1.772(1.346-2.333)$ & 0.027 & $1.415(1.041-1.923)$ \\
\hline CD44 expression (high vs. low) & 0.022 & $1.692(1.078-2.656)$ & 0.054 & $1.576(0.992-2.503)$ \\
\hline CD133 expression (high vs. low) & 0.005 & $1.676(1.170-2.401)$ & 0.153 & $1.314(0.903-1.912)$ \\
\hline Gender (male vs. female) & 0.718 & $0.942(0.682-1.301)$ & 0.884 & $1.025(0.732-1.437)$ \\
\hline Higher pathological grade & $<0.001$ & $1.466(1.197-1.795)$ & 0.004 & $1.389(1.108-1.741)$ \\
\hline Tumor size (>5 vs. $\leq 5 \mathrm{~cm}$ ) & 0.322 & $1.144(0.876-1.493)$ & 0.427 & $0.877(0.634-1.213)$ \\
\hline LYN (yes vs. no) & $<0.001$ & $1.749(1.338-2.286)$ & 0.003 & $1.783(1.217-2.612)$ \\
\hline
\end{tabular}

$P$ value $<0.05$ was considered significant. DFS, disease-free survival; HR, hazard ratio; ITGA7, integrin- $\alpha$; LYN, lymph node metastasis.

numerically reduced compared with the HR values of ITGA7 high group vs. ITGA7 low expression group. These data indicated that ITGA7/CD133 combination showed no better predicting value for DFS and OS compared to ITGA7 alone.

\section{Factors affecting DFS by Cox's proportional hazard model regression analyses}

Univariate Cox's regression analysis presented that ITGA7 high expression $(\mathrm{P}<0.001)$, $\mathrm{CD} 44$ high expression $(\mathrm{P}=0.022)$, $\mathrm{CD} 133$ high expression $(\mathrm{P}=0.005)$, higher pathological grade $(\mathrm{P}<0.001), L Y N(\mathrm{P}<0.001)$ and higher $T N M$ stage $(\mathrm{P}=0.002)$ were associated with lower DFS in NSCLC patients (Table 4). And further multivariate Cox's regression analysis indicated that ITGA7 high expression $(\mathrm{P}=0.027)$, higher pathological grade $(\mathrm{P}=0.004)$ and $\mathrm{LYN}(\mathrm{P}=0.003)$ were independent risk factors for DFS in NSCLC patients.

\section{Factors affecting OS by Cox's proportional hazard model regression analyses}

Univariate Cox's regression analysis revealed that ITGA7 high expression $(\mathrm{P}<0.001)$, $\mathrm{CD} 44$ high expression $(\mathrm{P}=0.045)$, CD133 high expression $(\mathrm{P}=0.010)$, higher pathological grade $(\mathrm{P}<0.001), \mathrm{LYN}(\mathrm{P}<0.001)$ and higher $\mathrm{TNM}$ stage $(\mathrm{P}=0.001)$ were correlated with reduced OS in NSCLC patients (Table 5). And multivariate Cox's regression analysis was further conducted, which indicated that ITGA7 high expression $(\mathrm{P}=0.006)$, higher pathological grade $(\mathrm{P}=0.006)$ and LYN $(\mathrm{P}<0.001)$ were independent risk factors for OS in NSCLC patients.

\section{Discussion}

In the present study, we observed that in NSCLC patients: (I) ITGA7 expression was positively associated with CD44 expression as well as CD133 expression; (II) ITGA7 was positively correlated with higher pathological grade, larger tumor size, LYN and higher TNM stage; (III) ITGA7 high expression was an independent risk factor for DFS and OS.

ITGA7 serves as the receptor for the extracellular matrix protein laminin, forms heterodimer with other integrins and is involved in several cellular processes (11). Recent studies reveal that ITGA7 acts as an oncogene and its overexpression promotes cell migration and invasion, reinforces epithelial-mesenchymal transition and enhances cell stemness in several cancers $(10,11)$. For example, ITGA7 promotes tumor initiation and metastasis but inhibits apoptosis, and high ITGA7 expression is associated with elevated expression of cancer stem cell markers including CD90, SOX2, NANOG etc. in esophageal squamous cell carcinoma (11). In another study, ITGA7 is shown to be a key functional receptor in glioblastoma stem cells and promotes AKT signaling pathway by inducing epithelial-mesenchymal transition as well as invasion of glioblastoma cells (10). In addition, CD44 and CD133, as common cancer stem cell markers, are reported to be involved in the NSCLC pathology and promote cell 
Table 5 Univariate and multivariate Cox's proportional hazard model regression analyses of factors affecting OS

\begin{tabular}{|c|c|c|c|c|}
\hline Items & \multicolumn{2}{|c|}{ Univariate Cox's regression } & \multicolumn{2}{|c|}{ Multivariate Cox's regression } \\
\hline ITGA7 expression (high vs. low) & $<0.001$ & $2.005(1.456-2.760)$ & 0.006 & $1.628(1.147-2.311)$ \\
\hline CD44 expression (high vs. low) & 0.045 & 1.717 (1.012-2.912) & 0.150 & $1.493(0.866-2.574)$ \\
\hline CD133 expression (high vs. low) & 0.010 & $1.656(1.131-2.426)$ & 0.222 & $1.281(0.861-1.908)$ \\
\hline Gender (male vs. female) & 0.725 & $0.938(0.657-1.340)$ & 0.941 & $1.014(0.701-1.467)$ \\
\hline Higher pathological grade & $<0.001$ & $1.522(1.216-1.906)$ & 0.006 & $1.428(1.106-1.844)$ \\
\hline Tumor size (>5 vs. $\leq 5 \mathrm{~cm})$ & 0.058 & $1.342(0.990-1.818)$ & 0.752 & $1.059(0.741-1.515)$ \\
\hline LYN (yes vs. no) & $<0.001$ & 2.268 (1.693-3.039) & $<0.001$ & $2.652(1.737-4.049)$ \\
\hline
\end{tabular}

$\mathrm{P}$ value $<0.05$ was considered significant. OS, overall survival; HR, hazard ratio; ITGA7, integrin- $\alpha$; LYN, lymph node metastasis.

proliferation as well as cell invasion $(9,13)$. In detail, CD44 overexpression is associated with advanced $\mathrm{T}$ stage as well as higher pathological grade and promotes tumor progression by elevating cell proliferation in NSCLC $(14,15)$. As for $\mathrm{CD} 133$, it is identified as a trans-membrane glycoprotein, which has been widely used as a marker to identify cancer stem cells in several cancers (13). There is evidence that overexpression of CD133 and CD44 promotes metastasis via WNT signaling pathway in $\operatorname{NSCLC~}(9,13,16)$. Therefore, we performed IHC assay to examine the expressions of ITGA7, CD44 and CD133, and to detect the correlation of ITGA7 with CD44 and CD133. We observed that ITGA7 expression was positively associated with CD44 and CD 133 expressions. The possible reasons might include that (I) ITGA7 overexpression might activate FAK/MARK signaling pathway, which might enhance the stemness of NSCLC cells and increase the expressions of cancer stem cell markers (CD44 and CD133). Conversely, overexpression of CD44 and CD133 might also activate the oncogenic pathway, which enhanced the expression of ITGA7, while the underlying mechanism needed exploration in further studies; (II) high ITGA7 expression might induce epithelial-mesenchymal transition, which might promote the cell invasion and metastasis in NSCLC, contributing to the activation of cancer cell stemness and the expression of cancer stem cell markers $(7,11)$.

Recent evidence referring to the correlation of ITGA7 with clinicopathological characteristics in human tumors, such as pathological grade, tumor size, has identified ITGA7 as an important promoter in tumor progression
$(10,11)$. For example, oesophageal squamous cell carcinoma patients with ITGA7 high expression present poor differentiation, further tumor invasion and advanced LYN (11). However, the correlation between ITGA7 expression and clinicopathological characteristics in NSCLC patients is still not investigated. In the present study, we found that ITGA7 high expression was correlated with higher pathological grade, larger tumor size, LYN and higher TNM stage. The possible reasons might be that: (I) ITGA7 high expression enhanced the stemness of cancer cells, which promoted the development and progression of NSCLC, leading to advanced TNM stage; (II) ITGA7 high expression activated oncogenic signaling pathway (such as: WNT signaling pathway) by triggering the overexpression of CD44, contributing to exacerbated metastasis and tumor progression, which resulted to advanced pathological grade (9).

There is limited research focusing on the correlation of ITGA7 expression with prognosis in cancers. Only one study exhibits that esophageal squamous cell carcinoma patients with ITGA7 high expression present shorter OS in comparison with those with ITGA7 low expression (11). Based on the previous data that ITGA7 high expression was associated with higher pathological grade as well as advanced TNM stage, and ITGA7 expression was positively correlated with the expressions of cancer stem cell markers, it was tempting to speculate that ITGA7 might be of predictive value for survival in NSCLC patients. And we observed that NSCLC patients with ITGA7 high expression had shorter DFS and OS compared to those 
with ITGA7 low expression and further Cox's proportional hazard model regression analyses exhibited that ITGA7 was an independent risk factor for DFS and OS in NSCLC patients. These could be explained by: (I) ITGA7 might enhance the stemness of cancer cells by upregulation of CD44 and CD133, which led to increased drug resistance and tumor metastasis, thus patients with ITGA7 high expression would response ineffectively to drugs and have worse prognosis; (II) ITGA7 was associated with higher pathological grade as well as advanced TNM stages, which were previously shown as risk factors for survival in NSCLC.

There existed some limitations in the current study: (I) this was a single-center study, therefore multiplecenter studies including more patients were necessary to validate the prognostic value of ITGA7; (II) the underlying mechanism of ITGA7 in NSCLC was not investigated in this study, thus further cellular experiments were needed; (III) we enrolled the NSCLC patients who underwent resection meanwhile did not receive neoadjuvant therapy in this study, whereas the role of ITGA7 in NSCLC patients who did not receive resection or who underwent neoadjuvant therapy was not investigated.

In conclusion, ITGA7 expression positively correlates with CD44 and CD133 expressions, and its high expression associates with advanced tumor features as well as poor survival in NSCLC patients, which suggests that ITGA7 may function as a potential prognostic biomarker for NSCLC and help with disease monitoring.

\section{Acknowledgments}

Funding: None.

\section{Footnote}

Conflicts of Interest: All authors have completed the ICMJE uniform disclosure form (available at http://dx.doi. org/10.21037/tcr.2019.08.31). The authors have no conflicts of interest to declare.

Ethical Statement: The authors are accountable for all aspects of the work in ensuring that questions related to the accuracy or integrity of any part of the work are appropriately investigated and resolved. The study was conducted in accordance with the Declaration of Helsinki (as revised in 2013). The present study was approved by the Ethics Committee of our hospital (approval number).
Written informed consents or verbal agreements with type recording were obtained from patients or their guardians.

Open Access Statement: This is an Open Access article distributed in accordance with the Creative Commons Attribution-NonCommercial-NoDerivs 4.0 International License (CC BY-NC-ND 4.0), which permits the noncommercial replication and distribution of the article with the strict proviso that no changes or edits are made and the original work is properly cited (including links to both the formal publication through the relevant DOI and the license). See: https://creativecommons.org/licenses/by-nc-nd/4.0/.

\section{References}

1. Bray F, Ferlay J, Soerjomataram I, et al. Global cancer statistics 2018: GLOBOCAN estimates of incidence and mortality worldwide for 36 cancers in 185 countries. CA Cancer J Clin 2018;68:394-424.

2. Watanabe SI, Nakagawa K, Suzuki K, et al. Neoadjuvant and adjuvant therapy for Stage III non-small cell lung cancer. Jpn J Clin Oncol 2017;47:1112-8.

3. Nagasaka M, Gadgeel SM. Role of chemotherapy and targeted therapy in early-stage non-small cell lung cancer. Expert Rev Anticancer Ther 2018;18:63-70.

4. Osmani L, Askin F, Gabrielson E, et al. Current WHO guidelines and the critical role of immunohistochemical markers in the subclassification of non-small cell lung carcinoma (NSCLC): Moving from targeted therapy to immunotherapy. Semin Cancer Biol 2018;52:103-9.

5. Zheng Y, de la Cruz CC, Sayles LC, et al. A rare population of CD24(+)ITGB4(+)Notch(hi) cells drives tumor propagation in NSCLC and requires Notch 3 for self-renewal. Cancer Cell 2013;24:59-74.

6. Desgrosellier JS, Cheresh DA. Integrins in cancer: biological implications and therapeutic opportunities. Nat Rev Cancer 2010;10:9-22.

7. Seguin L, Desgrosellier JS, Weis SM, et al. Integrins and cancer: regulators of cancer stemness, metastasis, and drug resistance. Trends Cell Biol 2015;25:234-40.

8. Diaz LK, Cristofanilli M, Zhou X, et al. Beta4 integrin subunit gene expression correlates with tumor size and nuclear grade in early breast cancer. Mod Pathol 2005;18:1165-75.

9. $\mathrm{Su} \mathrm{J}, \mathrm{Wu} \mathrm{S}, \mathrm{Wu} \mathrm{H}$, et al. CD44 is functionally crucial for driving lung cancer stem cells metastasis through Wnt/ beta-catenin-FoxM1-Twist signaling. Mol Carcinog 2016;55:1962-73. 
10. Haas TL, Sciuto MR, Brunetto L, et al. Integrin alpha7 Is a Functional Marker and Potential Therapeutic Target in Glioblastoma. Cell Stem Cell 2017;21:35-50.e9.

11. Ming XY, Fu L, Zhang LY, et al. Integrin alpha7 is a functional cancer stem cell surface marker in oesophageal squamous cell carcinoma. Nat Commun 2016;7:13568.

12. Ye SL, Li XY, Zhao K, et al. High expression of CD8 predicts favorable prognosis in patients with lung adenocarcinoma: A cohort study. Medicine (Baltimore) 2017;96:e6472.

13. Chen E, Zeng Z, Bai B, et al. The prognostic value of CSCs biomarker CD133 in NSCLC: a meta-analysis.

Cite this article as: Shi X, Wen F, Xu G, Hu Y. Integrin- $\alpha$ 7 expression positively correlates with CD44 and CD133 expressions, and its high expression associates with advanced tumor features as well as poor survivals in non-small cell lung cancer patients. Transl Cancer Res 2019;8(5):1806-1816. doi: 10.21037/tcr.2019.08.31
Oncotarget 2016;7:56526-39.

14. Hu B, Ma Y, Yang Y, et al. CD44 promotes cell proliferation in non-small cell lung cancer. Oncol Lett 2018;15:5627-33.

15. Roudi R, Madjd Z, Korourian A, et al. Clinical significance of putative cancer stem cell marker CD44 in different histological subtypes of lung cancer. Cancer Biomark 2014;14:457-67.

16. Qu H, Li R, Liu Z, et al. Prognostic value of cancer stem cell marker CD133 expression in non-small cell lung cancer: a systematic review. Int J Clin Exp Pathol 2013;6:2644-50. 